\title{
Electroaffinity Labeling: A New Platform for Chemoproteomic-based Target Identification
}

Authors: Yu Kawamata $\dagger^{1}$, Keun Ah Ryu $\dagger^{2}$, Gary N. Hermann $\dagger^{1}$, Alexander Sandahl ${ }^{1}$, Julien C. Vantourout $^{1}$, Aleksandra K. Olow ${ }^{3}$, La-Tonya A. Adams ${ }^{4}$, Eva Rivera-Chao ${ }^{1}$, Lee R. Roberts ${ }^{2}$, Rob C. Oslund*2, Olugbeminiyi O. Fadeyi*2, Phil S. Baran*

\author{
Affiliations: \\ ${ }^{1}$ Department of Chemistry, The Scripps Research Institute, 10550 North Torrey Pines Road, La \\ Jolla, CA 92037, USA. \\ ${ }^{2}$ Exploratory Science Center, Merck \& Co., Inc., Cambridge, MA 02141, USA. \\ ${ }^{3}$ Genetics and Pharmacogenomics, Merck \& Co., Inc., South San Francisco, CA 94080, USA. \\ ${ }^{4}$ Discovery Immunology, Merck \& Co., Inc., Cambridge, MA 02115, USA. \\ *Correspondence to: rob.oslund@merck.com, olugbeminiyi.fadeyi@merck.com, \\ pbaran@scripps.edu \\ $\dagger$ These authors contributed equally to this work.
}

\begin{abstract}
:
Target identification is a critical pillar within the drug discovery process that involves deconvoluting the protein target of a pharmacologically active small molecule ligand. While photoaffinity labeling strategies have become the benchmark for target deconvolution of small molecules owing to their reliance on external activation to induce covalent protein capture, the process of target identification remains one of the most technically challenging aspects of early drug discovery. Thus, there is a strong demand for new technologies that allow for controlled activation of chemical probes to covalently label their protein target. Here, we introduce an electroaffinity labeling (ECAL) platform which leverages the use of a small, redox-active diazetidinone (DZE) functional group to enable chemoproteomic-based target identification of pharmacophores within live cell environments.
\end{abstract}

\section{MAIN TEXT:}

\section{[Introduction]}

Improving the quality of target selection in the drug discovery process is the single most important factor to reducing drug attrition in the clinic. ${ }^{1-4}$ The selection of potential drug targets is aided by target identification and validation efforts that link a biologically active pharmacophore directly to the protein(s) it modulates. However, while the search for bioactive pharmacophores have been greatly enabled by disease relevant phenotypic assay screens, downstream identification of the actual protein target remains a challenging technical hurdle.,3,5 This hinders our ability to deconvolute therapeutically relevant targets and their involvement in disease biology. Thus, technologies that can facilitate the detection of a pharmacophore binding target within complex 
biological environments, such as the use of covalent capture methods, are in high demand to expedite commonly used downstream isolation and proteomic-based analytical efforts to determine target protein identity (Figure 1A).

Conventional affinity-based covalent capture technologies rely on the use of chemical- or photochemical-based cross-linker groups that, when appended to a pharmacophore of interest, enhance identification of their binding targets and molecular mechanisms (Figure 1B) ${ }^{5,6}$ In particular, photoaffinitiy labeling (PAL) through use of the diazirine, a unique three-membered ring containing $\mathrm{N}=\mathrm{N}$ bond, has emerged as a highly useful option due to its small size and high reactivity (Figure 1B) $\cdot^{7-10}$ Comparatively, the relatively inert nature of diazirine until photoactivation makes it far preferrable to chemical cross-linkers that are always activated and cause background labeling issues. However, despite its widespread adoption, the activation of diazirines relies on the use of high energy UV light, generating multiple reactive moieties such as carbenes and diazo species with differing bio-reactivity profiles that can complicate downstream proteomic analysis and target identification. ${ }^{11-13}$

Herein we present an electroaffinity labeling ("ECAL") platform technology in live cells (Figure 1C). The method relies on a similarly small functional group rarely explored in organic synthesis, a diazetidinone (DZE), that enables chemoproteomic-based target identification of pharmacophores within complex cell environments (Figure 1C). To showcase ECAL, we profiled DZE analogs of well-validated ligands as well as a previously unexplored REV-ERB $\alpha$ [also known as NR1D1 (nuclear receptor subfamily 1 group D member 1)] agonist in live cells. Recent studies have revealed REV-ERB $\alpha$ as a potential drug target due to its broad role in pathologies such as sleep disorder, metabolic syndrome, heart failure, cancer, and inflammation. ${ }^{14,15}$ Its putative ligands, ${ }^{16}$ SR-9009 and SR9011, have been utilized to elicit various pharmacological activities in

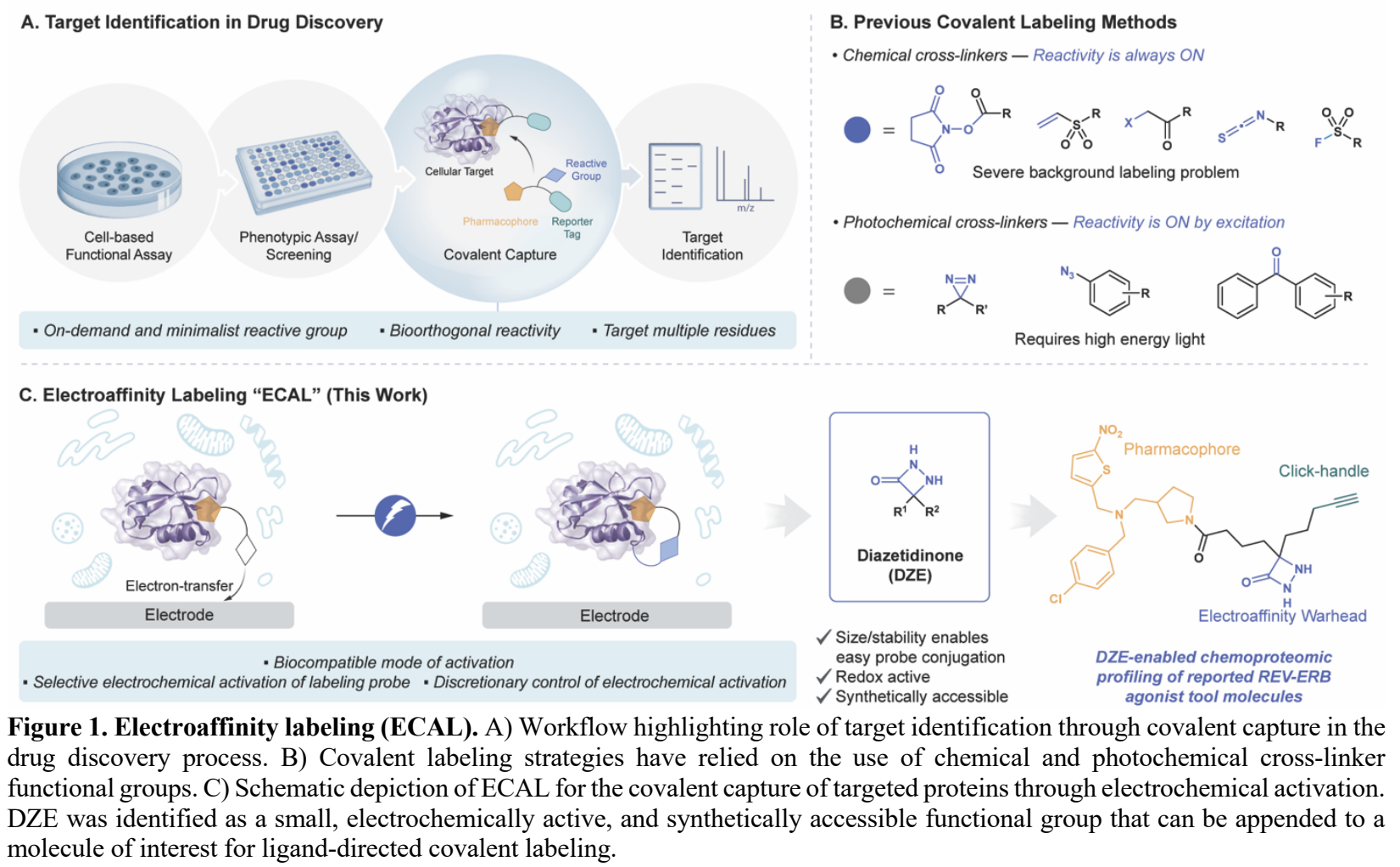

molecule of interest for ligand-directed covalent labeling. 
multiple in vitro and in vivo disease-phenotypes. ${ }^{15}$ However, despite the wide-ranging biological implications, chemoproteomic validation of REV-ERB $\alpha$ as the target for these ligands has not been reported.

\section{[Development of an electrochemically active functional group suitable for ECAL]}

As with photochemistry, electrochemistry is one of the oldest methods for facilitating redox reactions, which still remains the most direct means for accomplishing oxidation and reduction. Presumably due to its heterogeneous nature and lack of protocols that reliably proceed in water, the application of electrochemistry to biomolecule functionalization in complex biological systems is limited. ${ }^{17}$ Several recent successful applications in this context rely on the anodic oxidation of triazolinediones, ${ }^{18}$ phenothiazines ${ }^{19}$ or $N$-oxyls ${ }^{20}$ to capture tyrosine or tryptophan residues in small oligopeptides and in vitro protein labeling. To our knowledge, there is no precedent for electrochemical protein labeling in complex cellular environments. To begin these studies, several criteria were set for a useful functional group for ECAL. To allow minimally invasive placement of a reactive group on drug binding motifs, ${ }^{9}$ the desired functional group must be as small as possible, redox-active, stable under physiological conditions, and readily accessible (Figure 2A). It must also be easily incorporated into a trifunctional monomer that includes orthogonally reactive functional groups such as an alkyne and a carboxylic acid to enable modular incorporation of desired small molecule ligands as well as reporter groups. Most importantly, a highly reactive species should be generated through mild electrochemical activation that can be performed bioorthogonally in a complex cellular environment.

Satisfying these criteria is challenging, since redox-active compounds are usually reactive or possess large $\pi$-systems, deviating from the ideal probe design. Moreover, the redox potential necessary for activation needs to be ideally within $\pm 1 \mathrm{~V}$ (vs $\mathrm{Ag} / \mathrm{AgCl}$ ), considering the cathodic limit by $\mathrm{H}_{2} \mathrm{O}$ reduction (around $-1 \mathrm{~V}$ ) and anodic limit by oxidatively labile amino acid residues (Trp $1.02 \mathrm{~V}$, Tyr $0.93 \mathrm{~V}) .{ }^{17}$ On top of these difficulties, generating highly reactive species which rivals to carbenes (reactive species from photochemical activation of diazirines) through electrochemical redox under aqueous conditions in a biorthogonal manner is a demanding requirement. Not surprisingly, there is no such small motif known in the literature to satisfy all of these criteria. Accordingly, our explorations began by surveying a wide range of redox-active small molecules that could generate reactive species (Figure 2A). Some of them were inspired by existing literature, and others were designed based on first principles. For example, reductive means of activation on structures 1-3 were hypothesized to lead to reactive species such as vinylidene carbenes, ${ }^{21}$ quinone methides, ${ }^{22}$ and carbenes, ${ }^{23}$ respectively. Unfortunately, these strategies were barely successful from the standpoint of stability, size, accessibility, and modularity. Oxidative modes of activation were also explored on structures 4-9 wherein reactive carbenes, diazonium, ${ }^{24}$ benzynes, ${ }^{25}$ sulfeniums, and acyl pyridiniums ${ }^{26}$ could potentially be formed. Yet, these precursors showed no promising reactivity in a model study and were not further optimized or explored due to similar issues facing the reductive approach. Nevertheless, the oxidative approach was further pursued considering the fact that reactive species generated under oxidative conditions would likely be electrophilic, suitable for targeting peptides or proteins in which reactive amino acid residues are usually nucleophilic. The lesson from these exploratory studies and literature precedent was that hydrazine-containing species might be a promising motif due to their ease of oxidation under mild conditions. 
Returning back to the drawing board, we considered the smallest functional group possible analogous to the diazirines enlisted for photoaffinity labeling. The insertion of a single carbonyl group into a diazirine with the reduction of $\mathrm{N}=\mathrm{N}$ double bond led to the proposal of the diazetidinone (DZE) 10 (Figure 2B). This small, strained ring system was designed based on the intuitive notion that the embedded hydrazine motif would be readily oxidized to release a ketene upon nitrogen extrusion. Indeed, it is predicted that thermolysis of analogous structure could lead to ketene formation by cycloreversion, ${ }^{27}$ further supporting the notion that DZEs are prone to fragment into ketenes upon suitable activation. Of note, DZEs are a rarely utilized functional group in organic synthesis. Their main use is as a bioisostere of $\beta$-lactams, ${ }^{28}$ and acylated variants have been identified as serine-selective probes. ${ }^{29}$ Many methods for their preparation have been reported, ${ }^{28}$ yet their value as intermediates in synthesis is underexplored, and the electrochemical

\section{A. Early exploration}

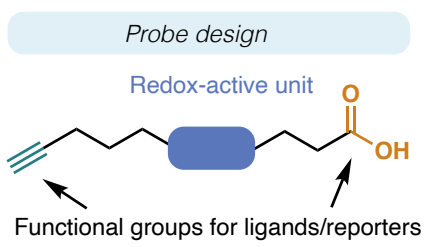

Criteria for redox-active unit

- Small and stable

$-1 \mathrm{~V}<$ redox potential $<1 \mathrm{~V}$ (vs Ag)

- Facile synthesis

- Generation of reactive intermediate

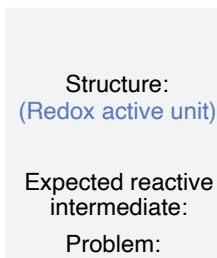

Reductive activation

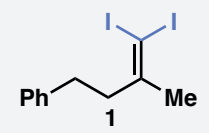

Vinylidene carbene

* Stability

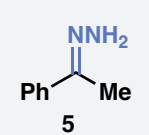

Diazo

* Accessibility

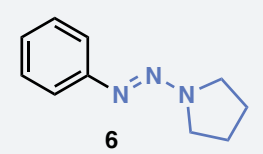

Diazonium

* Size

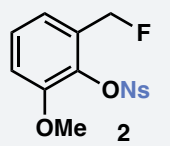

Quinone methide * Size

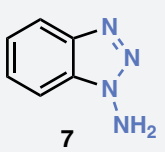

Benzyne

* Accessibility
Oxidative activation

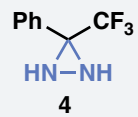

Carbene/diazo * Reactivity

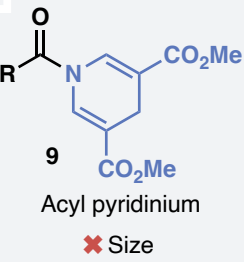

\section{B. Diazetidinone}

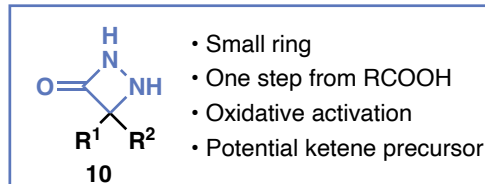

$$
\text { Mechanism of action }
$$

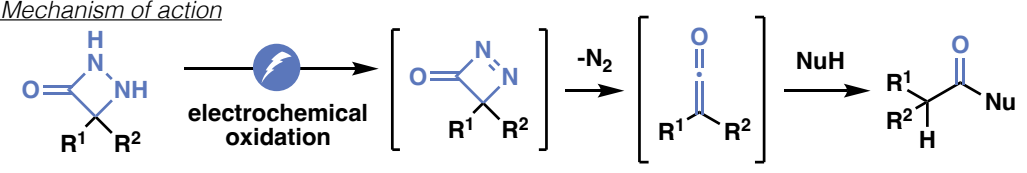

Proof of concept<smiles>NNC1(C(N)=O)CCCCC1</smiles>

11
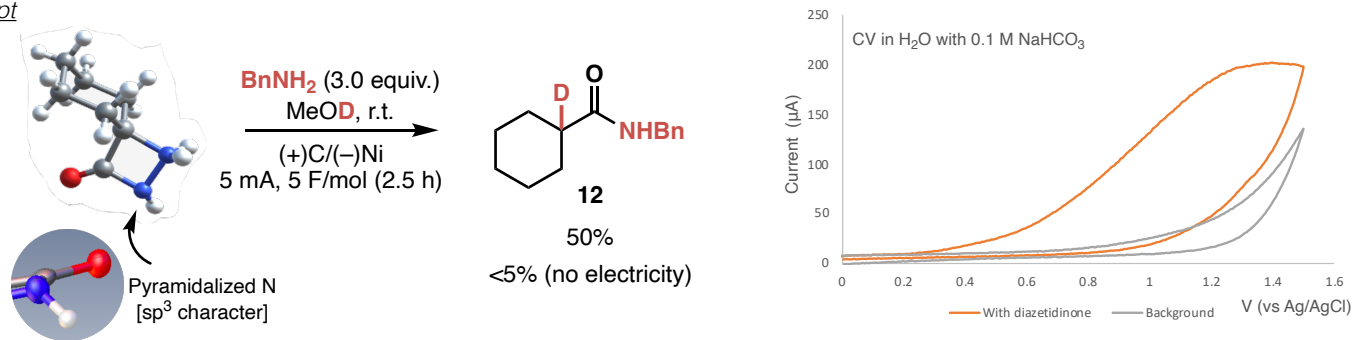

\section{General synthetic route}

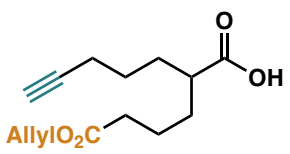

13
(1) $(\mathrm{COCl})_{2} \mathrm{CH}_{2} \mathrm{Cl}_{2} ; \mathrm{Et}_{3} \mathrm{~N}$; BocN=NBoc, DMAP, $46 \%$

(2) cat. $\operatorname{Pd}(0), 68 \%$

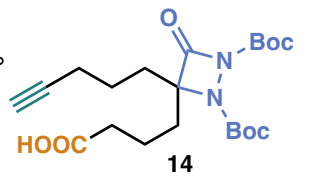

14

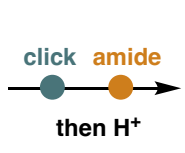

then $\mathrm{H}^{+}$

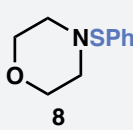

Sulfenium * Stability

3 steps from di-t-butylmalonate

Figure 2. Development of a redox-active small molecule for ECAL. A) Early exploration of various redox-active units to identify a small and reactive species that can be generated upon mild electrochemical activation. B) Diazetidinone and its electrochemical behavior. C) Synthesis of functional probes. 
behavior of DZEs is not reported. Thus, the model DZE 11 was prepared, and detailed analysis of 11 was conducted by X-ray crystallography as well as cyclic voltammetry (CV) (Figure 2B, Figure S1-S2). Interestingly, X-ray crystallographic analysis revealed that the amide nitrogen is considerably pyramidalized due to the highly strained four-membered ring, a rare example of twisted amides. ${ }^{30}$ Furthermore, a cyclic voltammetric study clearly suggested that $\mathbf{1 1}$ is indeed redox-active, although the oxidation peak around $1 \mathrm{~V}$ is ill-defined under the cyclic voltammetric conditions. Bulk electrolysis of $\mathbf{1 1}$ in the presence of benzylamine afforded an amide product $\mathbf{1 2}$ in moderate yield without significant oxidation of benzylamine, suggesting compatibility to reactive nucleophilic residues in peptides and proteins. Only a trace amount of $\mathbf{1 2}$ was observable without electrolysis, indicating that $\mathbf{1 1}$ is mostly unreactive during the timescale of the control experiment $(2.5 \mathrm{~h})$. The formation of amides as well as deuterium incorporation at the $\alpha$-position to the carbonyl support the hypothesis of a ketene as the reactive intermediate. Having demonstrated electrochemical activation of this latent functional group, we next designed a minimalist trifunctional monomer (14) consisting of a carboxylic acid group for ligand attachment, a reporter handle, and a DZE warhead. ${ }^{9}$ The key DZE intermediate 14 can be conveniently accessed from the carboxylic acid $\mathbf{1 3}$ in one step by [2+2] cycloaddition between the corresponding ketene and di-t-butyl azodicarboxylate (Figure 2C). ${ }^{31}$ Amide bond formations and click reactions can be used for attaching various ligands and reporter tags as exemplified through the synthesis of nine small molecule ligand-based DZE probes (see SI for complete listing).

\section{[Evaluation of DZE for targeted protein labeling in biologically compatible environments]}

With the DZE probes in hand, we first evaluated if a carbonic anhydrase(CA)-DZE-desthiobiotin trifunctional probe (sulfa-DZE, 15) can label free CA in an electrochemically dependent and selective manner. Accordingly, 15 was incubated in the presence of 1:1 CA and bovine serum albumin (BSA) in PBS followed by 1-minute electrolysis (Figure 3A). Visualization via western blot revealed desthiobiotinylation of $\mathrm{CA}$ in an electrochemically dependent manner with highly selective labeling of CA over BSA (Figure 3B and Figure S3). Importantly, this biotinylation effect could be competed away with free CA-inhibitor.

Next, the covalent labeling nature of the DZE probe was explored by performing peptide mapping of CA after electrolysis with 15. LC-MS/MS analysis showed modification of a diverse set of amino acids surrounding the entrance of the zinc binding pocket that include multiple aspartate, glutamate, and lysine residues, as well as minimally detected labeling of valine, glycine and phenylalanine (Figure 3C). As a comparison, photoaffinity labeling (PAL) was performed using a broadly utilized, fully functionalized dialkyl diazirine probe containing design features similar to our DZE probe 15. UV irradiation of the CA-diazirine probe $\mathbf{1 6}$ in the presence of CA resulted in labeling near the active site entrance similar to the DZE probe but with different residue labeling patterns that primarily includes glutamate and aspartate residues (Figure 3C). This carboxylate side chain preference achieved by $\mathbf{1 6}$ was consistent with recent studies using a dialkyl diazirine probe, ${ }^{11,12}$ highlighting the restricted amino acid residue reactivity profile for this commonly used PAL reagent. Thus, while both probes label within similar regions of the targeted protein, the broader amino acid labeling coverage achieved by the DZE probe facilitates increased opportunities for covalent protein capture within complex environments.

As further demonstration of the versatility of this electrochemical labeling approach, four additional small molecule ligand scaffolds (dasatinib, VX680, JQ-1, and PD0325901) were 

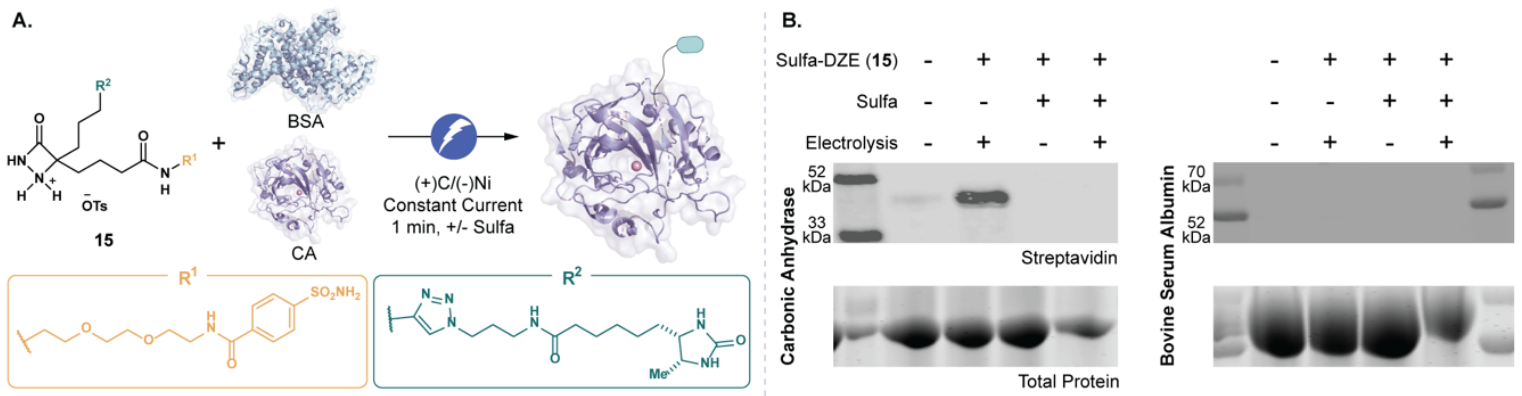

c.
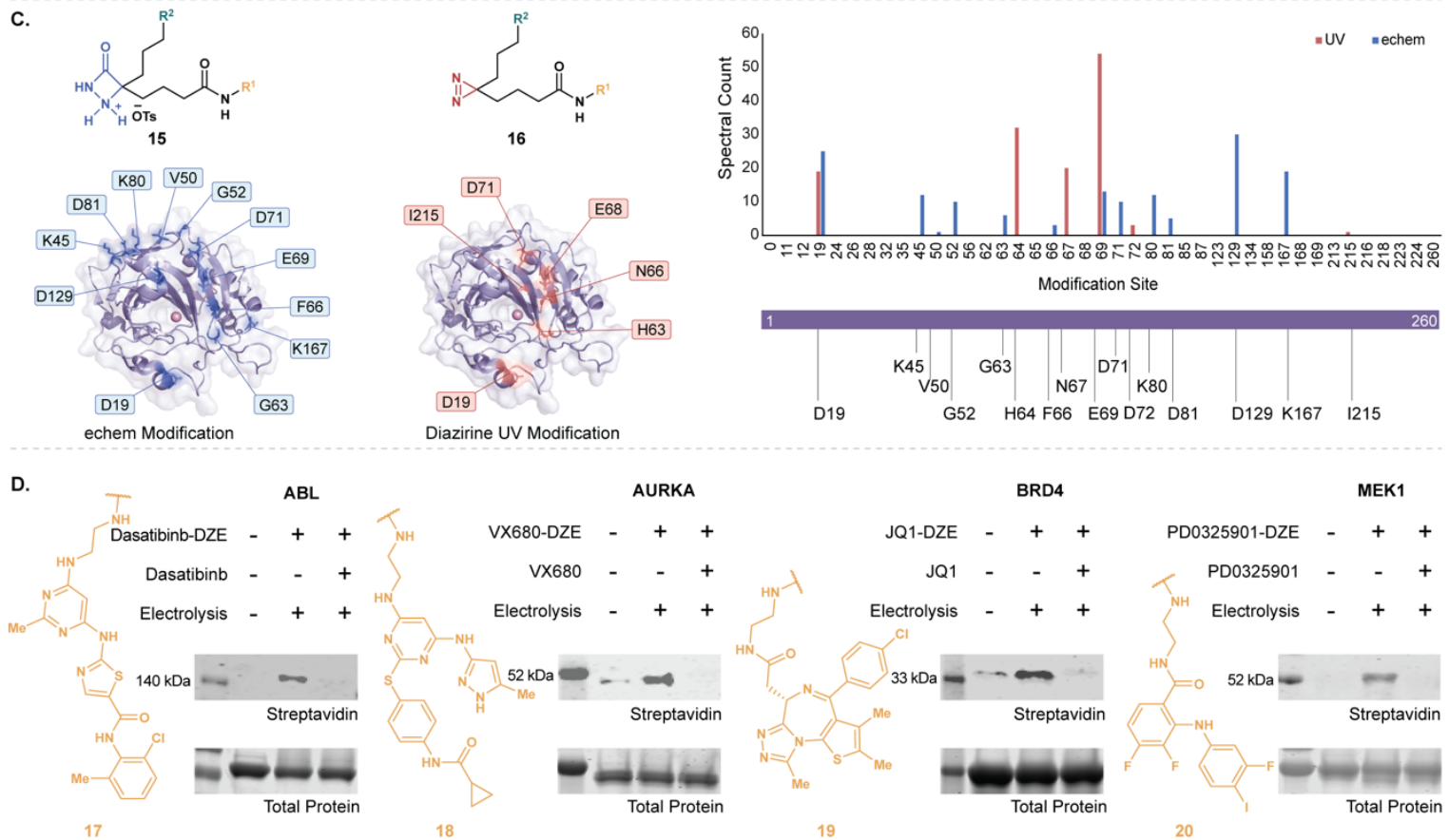

Figure 3. In vitro covalent capture of proteins using ECAL. A) Schematic depiction of Carbonic Anhydrase (CA)-targeted labeling using a DZE-containing arylsulfonamide probe (Sulfa-DZE, 15) in the presence or absence of Bovine Serum Albumin (BSA) bystander protein. B) Western blot analysis of targeted labeling in panel A. Desthiobiotinylation of CA was observed in the presence of electrolysis and could be competed away with excess free sulfonamide CA inhibitor (Sulfa). Desthiobiotinylation of BSA was not detected in any of the conditions. C) LC-MS/MS-based peptide mapping of CA labeled with a Sulfa-DZE (15) or Sulfa-Diazirine (16) using 1 min of electrochemical- or UV-activation, respectively. Labeled residues are highlighted on the protein and by bar plot analysis that shows residue number (x-axis) and spectral counts (y-axis) of labeled peptide containing the modification. D) Electroaffinity labeling of ABL, AURKA, BRD4, and MEK1 proteins using known small molecule ligands linked with DZE. Western blot analysis shows electrochemically dependent desthiobiotinylation that can be competed away with excess free ligand.

studied. ${ }^{9,32}$ The DZE functional group was appended to each of the inhibitors to prepare 17-20, and subsequently incubated in the presence of their respective binding proteins ABL, AURKA, BRD4, and MEK1. Electrochemically dependent desthiobiotinylation was detected for each of these protein/DZE-ligand pairs that could be competed away in the presence of excess free ligand (Figure 3D). Importantly, similar to the CA labeling experiment in Figure 3B, the labeling effect was specific to the target protein as no off-target protein labeling was observed in the presence of a bystander protein (Figure S4). Additionally, to investigate the ligand compatibility of our ECAL 
system with other targeting modalities, the DZE tri-functional monomer was conjugated to a COV2-Spike binding peptide ${ }^{33}$ and observed ligand-dependent labeling of the Spike protein (Figure S5).

\section{[ECAL in live cells]}

Having demonstrated the compatibility of ECAL with a wide range of ligand-protein pairs, ECAL was field-tested within the context of chemoproteomic profiling of known ligands in live cell culture. Considering that this is the first instance of ECAL in live cell systems, the biocompatibility of the electrochemical system on cell viability was evaluated with the finding that short reaction times employed within our labeling experiments do not impact cell viability (Figure S6). Using dasatinib or JQ1 equipped with DZE and desthiobiotin (compounds 17 or 19) we set out to identify endogenous proteins labeled within HCT116 colorectal cancer cells (Figure 4A). Cells were incubated with the DZE probes followed by electrochemical activation to initiate covalent protein labeling followed by enrichment for subsequent proteomic analysis (Figure 4B). Targeted labeling using JQ1-DZE-desthiobiotin probe 19 in HCT116 cells resulted in the significant enrichment of the known binding partners BRD4 and BRD2 (Figure 4C). In contrast, when Dasatinib-DZEdesthiobiotin 17 was used for targeted protein labeling, SRC kinase (a known dasatinib ligand) was identified as a highly enriched target (Figure 4D). Comparing the 25 most enriched proteins detected from JQ1-DZE-desthiobiotin 19 and Dasatinib-DZE-desthiobiotin 17 targeted labeling led to no observed direct protein overlap (Figure 3E) as well as distinct differences in enriched GO-terms for biological and molecular functions (Figure 3F and 3G). The generality of our ECAL technology was further showcased through CA-DZE 15 targeted labeling of CA in HT29 cells that resulted in increased CA enrichment in both hypoxic and normoxic conditions (Figure S7). ${ }^{34}$ These results highlight the ability of the ECAL technology to achieve selective chemoproteomic detection of ligand targets in complex biological systems (Figure S8-9). Regarding how the labeling of endogenous proteins was achieved using an exterior electrode, at this moment we consider that both direct and mediated oxidation pathways are possible. It is known that direct exchange of electrons is possible through cell membranes such as the case in red blood cells. ${ }^{35} \mathrm{On}$ the other hand, mediated oxidation is also feasible considering the myriad of redox-active cofactors as well as redox-active proteins that participate within various electron-transfer pathways in and through a cell. ${ }^{36}$ However, unveiling the detailed mechanism of the probe activation is outside the focus of the current study, and could be a subject of future investigation.

\section{[ECAL for chemoproteomic profiling REV-ERB ligands]}

Having established our ECAL technology as a robust approach for target identification, chemoproteomic profiling of two structurally similar tertiary amine (TA) pharmacophores, SR9009 and SR9011 was pursued. ${ }^{15}$ These compounds were initially developed at Scripps as synthetic agonists of transcription factors REV-ERB $\alpha$ and REV-ERB $\beta$ for the regulation of circadian rhythm, ${ }^{16}$ and have attracted further interest for their involvement in a number of important cell functions through suspected REV-ERB interactions. ${ }^{15}$ This is due, in part, to the connection of REV-ERB to biological processes under control of the circadian cycle that includes immunological and metabolic pathways. ${ }^{37,38}$ In an in vivo study, cellular treatment with SR9009 


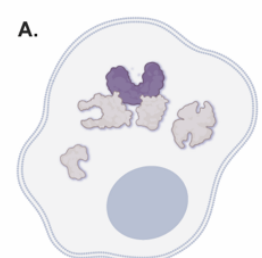

Live Cell Drug-DZE Incubation

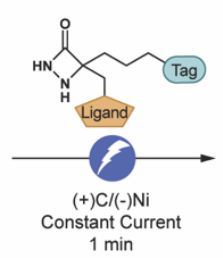

$1 \mathrm{~min}$

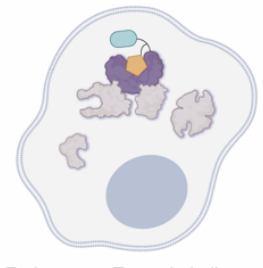

Endogenous Target Labeling

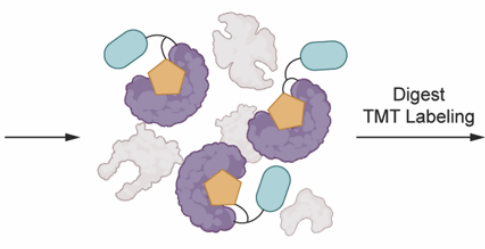

Cell lysis and biotin enrichment

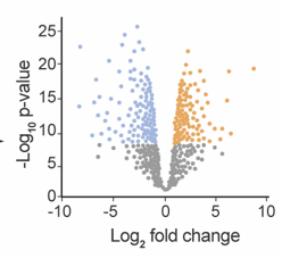

Quantitative MS Identification

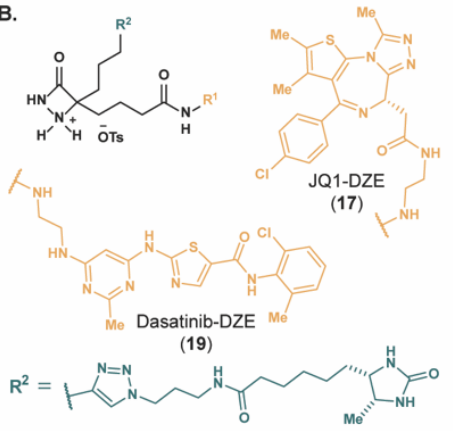

C.

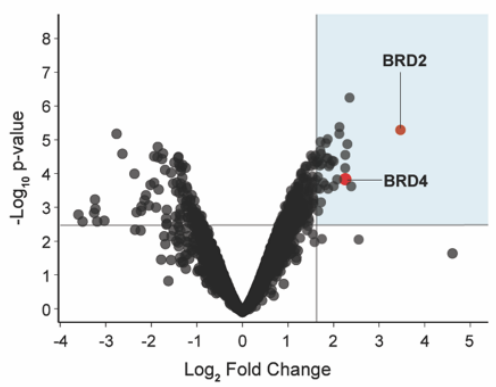

E.

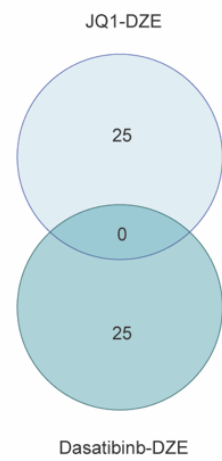

F.

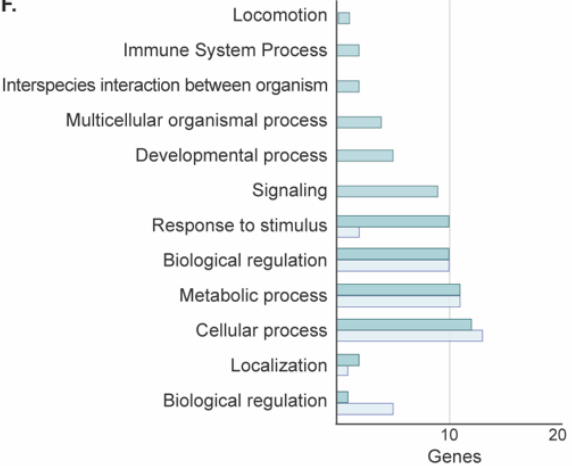

D.

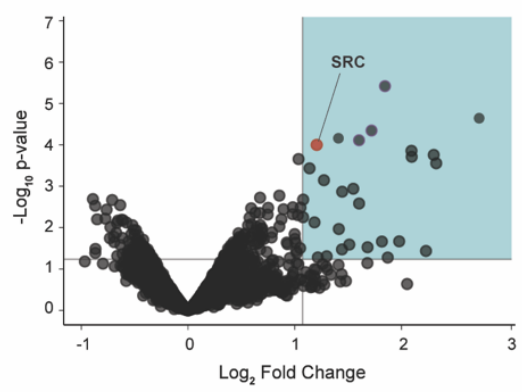

G.

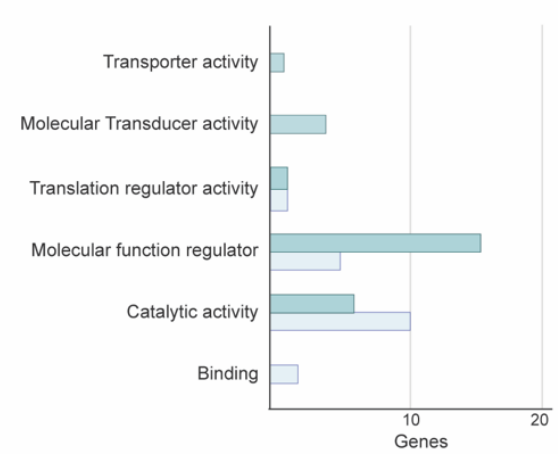

G.

Figure 4. Targeted protein labeling in live cells using ECAL. A) Schematic depiction of live cell labeling via ECAL using a DZE-based ligand followed by LC-MS/MS analysis for protein identification. B) JQ1-DZE (19) or Dasatinib-DZE (17) probes used for targeted labeling in HCT116 cells. C) Volcano plot analysis of HCT116 cells treated with JQ1-DZE with or without excess free JQ1 followed by ECAL (1 min) resulting in enrichment of known JQ1 protein targets (BRD2, BRD4, red dots). D) Volcano plot analysis of HCT116 cells treated with Dasatinib-DZE with or without excess free Dasatinib followed by ECAL (1 min) resulting in enrichment of a known Dasatinib protein target (SRC, red dot). E) Venn diagram analysis of top 25 enriched proteins from JQ1-DZE (light blue) or Dasatinib-DZE (dark blue) targeted labeling within the same cell line (HCT116) shows no overlap of enriched proteins. Gene ontology term analysis of F) biological and G) molecular functions for protein hits enriched from JQ-1DZE (light blue) and Dasatinib-DZE (dark blue) targeting.

was linked to REV-ERB $\alpha$-mediated boosting of exercise capacity via increased mitochondrial production in skeletal muscle. ${ }^{39}$ This observation has led to the illicit usage of these SR9009-based agonists as exercise supplements and performance-enhancers within the body-building community. ${ }^{40}$ SR 9009 and SR9011 have also shown the ability to effect other biological processes that include the negative regulation of proinflammatory Th17 cell-mediated autoimmunity ${ }^{41}$ and tumor cell killing, ${ }^{42}$ all presumably through REV-ERB modulation. However, despite the illicit use in humans and the multiple in vitro and in vivo based activities of SR9009 and SR9011, direct identification of REV-ERB as the protein target is lacking. Thus, we set out to validate REV-ERB 
binding of these important biologically relevant molecules via chemoproteomic analysis using our ECAL technology.

TA-DZE probe $\mathbf{2 1}$ was rationally designed based on our minimalist trifunctional monomer 14 and the required TA pharmacophore of the SR9009 agonist (Figure 5B). Amide coupling of SR9009 synthetic agonist core with DZE trifunctional monomer 14 followed by Boc-deprotection delivered the desired probe $\mathbf{2 1}$ in high yields (49\% for two steps). To confirm that the newly designed and synthesized TA-DZE probe $\mathbf{2 1}$ retains desired pharmacological properties, the probe was screened along with parent compounds SR9009 and SR9011 in a previously reported Th17 differentiation assay on primary cells. ${ }^{41}$ Primary human CD4 T cells were cultured under Th17 differentiation conditions with or without compounds (DMSO, SR9009, SR9011 and TA-DZE probe 21). Similar to parent compounds SR9009 and SR9011, TA-DZE probe 21 treatment inhibits Th17 differentiation (Figure 5C).

With the desired TA-DZE probe in hand, chemoproteomic analysis using ECAL was performed in HEK293T cells overexpressing REV-ERB $\alpha$, the reported target of this molecular scaffold (Figure 5D and Figure S10). Accordingly, HEK293T-REV-ERB $\alpha$ cells were incubated with TADZE probe 21 with or without electrolysis. Following the electrochemical treatment step, labeled proteins were then conjugated to biotin-azide through $\mathrm{CuAAC}$ click reaction and affinity purified on streptavidin beads. Enriched proteins were subjected to tryptic digestion and downstream TMTbased quantitative LC-MS/MS analysis. Volcano plot analysis identified proteins from our ECAL method showing significant enrichment over the no electrolysis controls (Figure 5E). Gene ontology (GO) analysis of the detected proteins showed enrichment of biological terms associated with both SR9009/SR9011 and REV-ERB activity that include metabolic and gene expression processes (Figure 5F). However, to our surprise, REV-ERB $\alpha$ was not enriched with our TA-DZE probe 21 despite overexpression of this protein in 293T cells (Figure 5E and Figure S11). This result, in conjunction with a recent study reporting that SR9009 modulates cellular metabolism, viability, proliferation, and gene expression in REV-ERB knockout systems, ${ }^{43}$ suggests that these TA scaffolds potentially operate independently of REV-ERB.

Given the broad pharmacological properties of these TA scaffolds and failure to detect REV-ERB protein enrichment, the gene expression pattern of protein hits from ECAL targeted labeling method across various cell types was studied using a single-cell RNA sequencing (scRNAseq) BioTuring Database comprising 255 gene studies. Through the analysis of ranked expression, we observed that majority of these genes are most prominently expressed within lymphoid, $\mathrm{T}$ and $\mathrm{B}$ cell populations (Figure 5G). In addition, a subset of 19 genes were detected that shared similar expression patterns across cell subsets to ROR $\gamma$ and REV-ERB $\alpha$ (NR1D1) suggesting shared function or biology (Figure S12). The reported role of SR9009 in modulating Th17 cell differentiation ${ }^{41}$ led us to next examine the gene expression profile of enriched protein hits within naïve CD4+ T cells and differentiated Th17 cells, as annotated in the BioTuring resource. From this analysis we identified genes that are abundantly and commonly expressed in cells representing Th17 and naïve CD4 T cells (>70\%), including FAU, YBX1, SUB1 and APRT (Figure 5H). We also observed some genes that are more abundantly found in Th17 cells as compared to naïve CD4 T cells (e.g. TPI1, PPIB, LYAR, ELOVL5 and GYG1). Collectively, these results suggest that the Th17 differentiation effects of these compounds potentially occur in a REV-ERB binding independent manner and further highlight their possible pleiotropic nature. 
A.

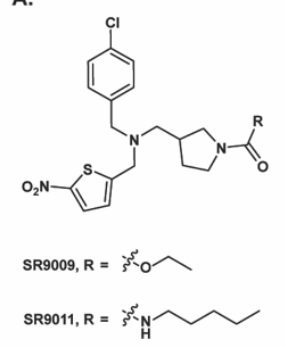

B.

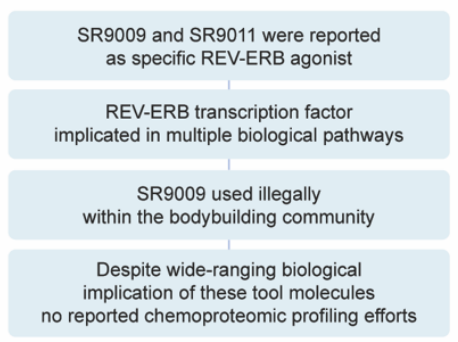

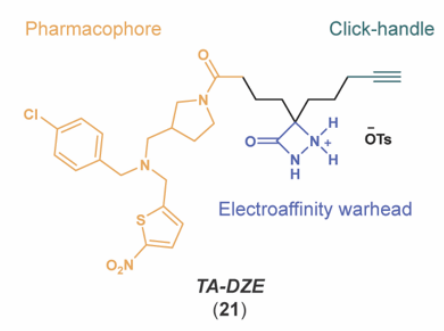

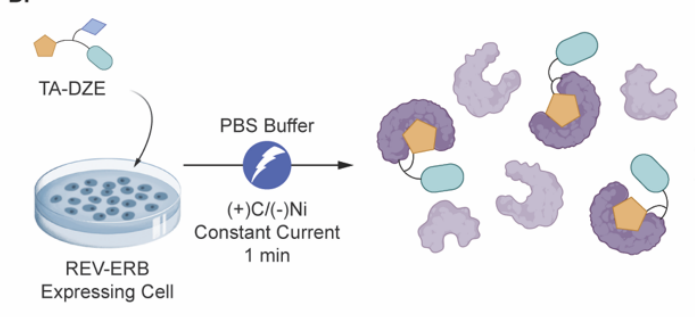

E.

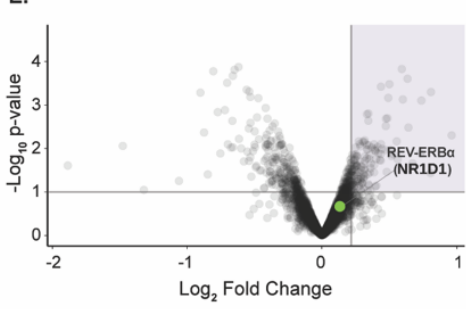

c.

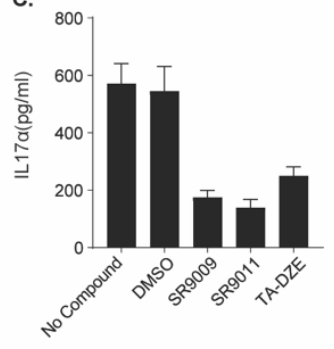

H.

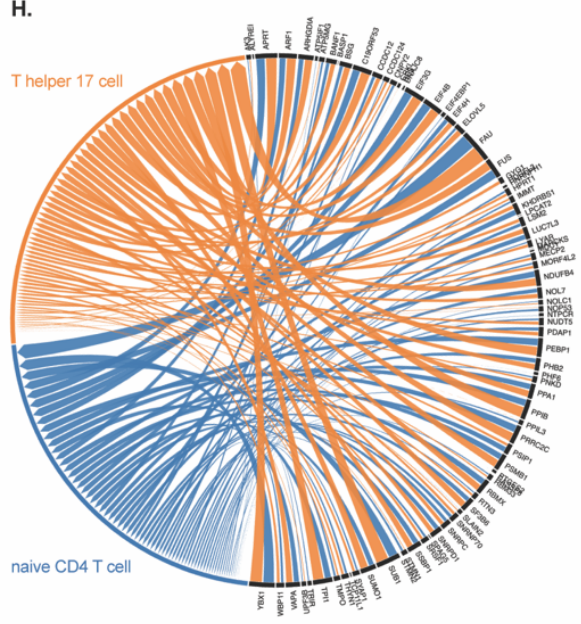

G.

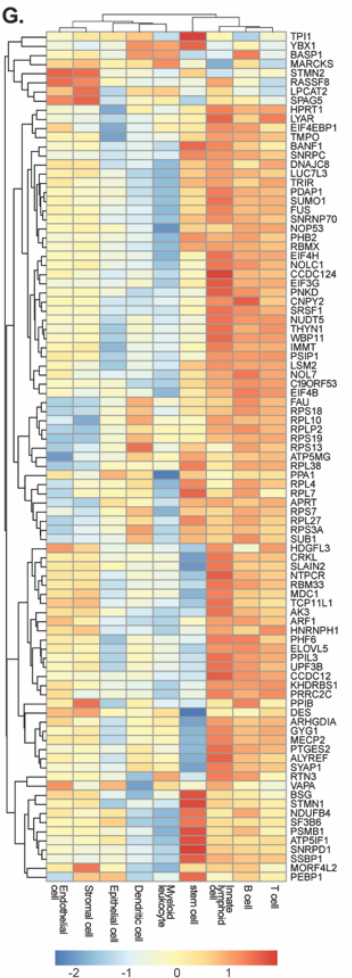

Figure 5. ECAL chemoproteomic profiling of SR9009-based DZE probe. A) Chemical structures of SR9009 and SR9011 tool compounds and description of their broad biological utilization. B) SR9009 and SR9011 ligand DZE probe design based on tertiary amine (TA) pharmacophore (TA-DZE, 21). C) Th17 cell differentiation assay showing functional activity of SR9009, SR9011, and TA-DZE. D) Schematic showing ECAL of 293T cells overexpressing REV-ERB $\alpha$ (NR1D1). E) Volcano plot analysis of HEK293T cells overexpressing REV-ERB $\alpha$ treated with TA-DZE with or without electrochemical activation (REV-ERB $\alpha$ (NR1D1), green dot). F) GO-term analysis of top 100 enriched protein hits combined from two independent experiments. G) Gene expression profile analysis of the top 100 enriched protein hits (combined from duplicate analysis) across a single cell RNA sequencing database (BioTuring) shown as a z-score heat map of genes (y-axis) across multiple cell types (x-axis). H) Circos plot of genes from the top 100 enriched protein hits (combined from duplicate analysis) across naïve CD4+ (blue) and Th17 (orange) T cells. Line width represents percentage of cells with expression of the indicated gene.

\section{[Conclusion]}

This disclosure reports the development of the first electrochemical-based targeted protein labeling platform within live cells. This technology relies on the use of a minimalist DZE functional group that leverages its intrinsic electrochemical activation properties to generate a reactive intermediate for covalent protein labeling. By appending appropriately functionalized DZE to small molecule ligands of interest, targeted labeling of proteins can be achieved in an electrochemically dependent fashion in both free protein and complex cellular environments. The biocompatibility and reaction 
tunability afforded by electrochemical activation will likely open up new possibilities for chemical biology-based applications within biological environments.

\section{ASSOCIATED CONTENT}

\section{Supporting Information}

Experimental procedures and characterizations of compounds.

\section{AUTHOR INFORMATION}

\section{Corresponding Author}

*pbaran@scripps.edu

*rob.oslund@merck.com,

*olugbeminiyi.fadeyi@merck.com

\section{Author Contributions}

RCO, OOF and PSB conceptualized the study, YK, GNH, AFS, JCV, ERC, PSB designed and performed chemical experiments, KAR, LAA, AKO, LRR, RCO, OOF designed and performed biological experiments. All the authors contributed to data analysis. YK, KAR, RCO, OOF, PSB wrote the manuscript.

\section{Notes}

KAR, LAA, AKO, LRR, RCO, and OOF are/were employees of Merck and Co., Inc. during the preparation of this manuscript.

\section{ACKNOWLEDGMENT}

The work was supported by National Science Foundation Center for Synthetic Organic Electrochemistry CHE-2002158, National Institutes of Health grant GM-118176, and gifts from Merck \& Co., Inc., Kenilworth, New Jersey, USA. G.N.H was funded by the Deutsche Forschungsgemeinschaft (DFG, German Research Foundation) - 419055018 / HE 8427/1-1. A.F.S. was supported by Lundbeck Foundation (Grant No. R208-2015-3354), E.R.-C. was supported by the Galician Programme for Research, Innovation and Growth for 2018. We thank D.-H. Huang and L. Pasternack (Scripps Research) for assistance with NMR spectroscopy; J. Chen, B. Sanchez, and E. Sturgell (Automated Synthesis Facility, Scripps Research) for purification of compounds and acquisition of HRMS data. We thank T. Wyche (Merck \& Co., Inc.) for assistance with HRMS data, S. Ingale (Merck \& Co., Inc.) for assistance with peptide synthesis, and J. Oh (Merck \& Co., Inc.) for helpful discussions.

\section{REFERENCES}

(1) Bunnage, M. E.; Chekler, E. L. P.; Jones, L. H. Target Validation Using Chemical Probes. Nat. Chem. Biol. 2013, 9 (4), 195-199.

(2) Emmerich, C. H.; Gamboa, L. M.; Hofmann, M. C. J.; Bonin-Andresen, M.; Arbach, O.; Schendel, P.; Gerlach, B.; Hempel, K.; Bespalov, A.; Dirnagl, U.; Parnham, M. J. Improving 
Target Assessment in Biomedical Research: The GOT-IT Recommendations. Nat. Rev. Drug Discov. 2021, 20 (1), 64-81.

(3) Kiriiri, G. K.; Njogu, P. M.; Mwangi, A. N. Exploring Different Approaches to Improve the Success of Drug Discovery and Development Projects: A Review. Future J. Pharm. Sci. 2020, 6 (1), 27.

(4) Bunnage, M. E. Getting Pharmaceutical R\&D Back on Target. Nat. Chem. Biol. 2011, 7 (6), $335-339$.

(5) Schenone, M.; Dančík, V.; Wagner, B. K.; Clemons, P. A. Target Identification and Mechanism of Action in Chemical Biology and Drug Discovery. Nat. Chem. Biol. 2013, 9 (4), 232-240.

(6) Park, J.; Koh, M.; Park, S. B. From Noncovalent to Covalent Bonds: A Paradigm Shift in Target Protein Identification. Mol. Biosyst. 2012, 9 (4), 544-550.

(7) Sumranjit, J.; Chung, S. J. Recent Advances in Target Characterization and Identification by Photoaffinity Probes. Molecules 2013, 18 (9), 10425-10451.

(8) Smith, E.; Collins, I. Photoaffinity Labeling in Target- and Binding-Site Identification.

Future Med. Chem. 2015, 7 (2), 159-183.

(9) Li, Z.; Hao, P.; Li, L.; Tan, C. Y. J.; Cheng, X.; Chen, G. Y. J.; Sze, S. K.; Shen, H.; Yao, S. Q. Design and Synthesis of Minimalist Terminal Alkyne-Containing Diazirine PhotoCrosslinkers and Their Incorporation into Kinase Inhibitors for Cell- and Tissue-Based Proteome Profiling . Angew. Chem. Int. Ed. 2013, 52 (33), 8551-8556.

(10) Murale, D. P.; Hong, S. C.; Haque, Md. M.; Lee, J.-S. Photo-Affinity Labeling (PAL) in Chemical Proteomics: A Handy Tool to Investigate Protein-Protein Interactions (PPIs). Proteome Sci. 2017, 15 (1), 14.

(11) West, A. V.; Muncipinto, G.; Wu, H.-Y.; Huang, A. C.; Labenski, M. T.; Jones, L. H.; Woo, C. M. Labeling Preferences of Diazirines with Protein Biomolecules. J. Am. Chem. Soc. 2021, 143 (17), 6691-6700.

(12) Conway, L. P.; Jadhav, A. M.; Homan, R. A.; Li, W.; Rubiano, J. S.; Hawkins, R.; Lawrence, R. M.; Parker, C. G. Evaluation of Fully-Functionalized Diazirine Tags for Chemical Proteomic Applications. Chem. Sci 2021, 12 (22), 7839-7847.

(13) Brunner, J. New Photolabeling and Crosslinking Methods. Annu. Rev. Biochem. 1993, 62 (1), 483-514.

(14) Kojetin, D. J.; Burris, T. P. REV-ERB and ROR Nuclear Receptors as Drug Targets. Nat. Rev. Drug Discov. 2014, 13 (3), 197-216. 
(15) Uriz-Huarte, A.; Date, A.; Ang, H.; Ali, S.; Brady, H. J. M.; Fuchter, M. J. The Transcriptional Repressor REV-ERB as a Novel Target for Disease. Bioorg. Med. Chem. Lett. 2020, 30 (17), 127395.

(16) Solt, L. A.; Wang, Y.; Banerjee, S.; Hughes, T.; Kojetin, D. J.; Lundasen, T.; Shin, Y.; Liu, J.; Cameron, M. D.; Noel, R.; Yoo, S.-H.; Takahashi, J. S.; Butler, A. A.; Kamenecka, T. M.; Burris, T. P. Regulation of Circadian Behavior and Metabolism by Synthetic REV-ERB Agonists. Nature 2012, 485 (7396), 62-68.

(17) Mackay, A. S.; Payne, R. J.; Malins, L. R. Electrochemistry for the Chemoselective Modification of Peptides and Proteins. J. Am. Chem. Soc. 2022, 144 (1), 23-41.

(18) Alvarez-Dorta, D.; Thobie-Gautier, C.; Croyal, M.; Bouzelha, M.; Mével, M.; Deniaud, D.; Boujtita, M.; Gouin, S. G. Electrochemically Promoted Tyrosine-Click-Chemistry for Protein Labeling. J. Am. Chem. Soc. 2018, 140 (49), 17120-17126.

(19) Song, C.; Liu, K.; Wang, Z.; Ding, B.; Wang, S.; Weng, Y.; Chiang, C.-W.; Lei, A. Electrochemical Oxidation Induced Selective Tyrosine Bioconjugation for the Modification of Biomolecules. Chem. Sci. 2019, 10 (34), 7982-7987.

(20) Toyama, E.; Marumaya, K.; Sugai, T.; Kondo, M.; Masaoka, S.; Saitoh, T.; Oisaki, K.; Kanai, M. Electrochemical Tryptophan-Selective Bioconjugation. https://doi.org/10.26434/chemrxiv.7795484.

(21) Stang, P. J. Unsaturated Carbenes. Chem. Rev. 1978, 78 (4), 383-405.

(22) Minard, A.; Liano, D.; Wang, X.; Antonio, M. D. The Unexplored Potential of Quinone Methides in Chemical Biology. Bioorgan. Med. Chem. 2019, 27 (12), 2298-2305.

(23) Tomioka, H.; Hayashi, N.; Izawa, Y.; Liu, M. T. H. Photolysis of 3-Chlorodiazirine in the Presence of Alkenes. Kinetic Evidence for Intervention of a Carbene-Alkene Intermediate in Addition of Chlorocarbene to Alkene. J. Am. Chem. Soc. 1984, 106 (2), 454-456.

(24) Voica, A.-F.; Mendoza, A.; Gutekunst, W. R.; Fraga, J. O.; Baran, P. S. Guided Desaturation of Unactivated Aliphatics. Na.t Chem. 2012, 4 (8), 629-635.

(25) Li, L.; Li, Y.; Fu, N.; Zhang, L.; Luo, S. Catalytic Asymmetric Electrochemical A-Arylation of Cyclic B-Ketocarbonyls with Anodic Benzyne Intermediates. Angew. Chem. Int. Ed. 2020, 59 (34), 14347-14351.

(26) Funder, E. D.; Trads, J. B.; Gothelf, K. V. Oxidative Activation of Dihydropyridine Amides to Reactive Acyl Donors. Org. Biomol. Chem. 2014, 13 (1), 185-198.

(27) Sterk, H.; Uray, G.; Ziegler, E. Über Einen Versuch Zur Berechnung Der Fragmentation von $\beta$-Lactamen Mittels Der EHT-Methode. Monatsh. Chem. 1972, 103 (2), 615-623. 
(28) Jungheim, L. N. Chapter Five The Chemistry of 1,2-Diazetidin-3-Ones. Adv. Heterocycl. Chem. 2013, 110, 145-174.

(29) Zuhl, A. M.; Mohr, J. T.; Bachovchin, D. A.; Niessen, S.; Hsu, K.-L.; Berlin, J. M.; Dochnahl, M.; López-Alberca, M. P.; Fu, G. C.; Cravatt, B. F. Competitive Activity-Based Protein Profiling Identifies Aza- $\beta$-Lactams as a Versatile Chemotype for Serine Hydrolase Inhibition. J. Am. Chem. Soc. 2012, 134 (11), 5068-5071.

(30) Liu, C.; Szostak, M. Twisted Amides: From Obscurity to Broadly Useful Transition-MetalCatalyzed Reactions by N-C Amide Bond Activation. Chem. Eur. J. 2017, 23 (30), 7157-7173.

(31) Berlin, J. M.; Fu, G. C. Enantioselective Nucleophilic Catalysis: The Synthesis of Aza- $\beta$ Lactams through [2+2] Cycloadditions of Ketenes with Azo Compounds. . Angew. Chem. Int. Ed. 2008, 47 (37), 7048-7050.

(32) Tyler, D. S.; Vappiani, J.; Cañeque, T.; Lam, E. Y. N.; Ward, A.; Gilan, O.; Chan, Y.-C.; Hienzsch, A.; Rutkowska, A.; Werner, T.; Wagner, A. J.; Lugo, D.; Gregory, R.; Molina, C. R.; Garton, N.; Wellaway, C. R.; Jackson, S.; MacPherson, L.; Figueiredo, M.; Stolzenburg, S.; Bell, C. C.; House, C.; Dawson, S.-J.; Hawkins, E. D.; Drewes, G.; Prinjha, R. K.; Rodriguez, R.; Grandi, P.; Dawson, M. A. Click Chemistry Enables Preclinical Evaluation of Targeted Epigenetic Therapies. Science 2017, 356 (6345), 1397-1401.

(33) Pomplun, S.; Jbara, M.; Quartararo, A. J.; Zhang, G.; Brown, J. S.; Lee, Y.-C.; Ye, X.; Hanna, S.; Pentelute, B. L. De Novo Discovery of High-Affinity Peptide Binders for the SARSCoV-2 Spike Protein. ACS Cent. Sci 2021, 7 (1), 156-163.

(34) Jeong, S.-H.; Jeon, Y.-J.; Park, S. J. Inhibitory Effects of Dieckol on Hypoxia-Induced Epithelial-Mesenchymal Transition of HT29 Human Colorectal Cancer Cells. Mol. Med. Rep. 2016, 14 (6), 5148-5154.

(35) Yu, C.; Wang, L.; Zhu, Z.; Bao, N.; Gu, H. Trans-Membrane Electron Transfer in Red Blood Cells Immobilized in a Chitosan Film on a Glassy Carbon Electrode. Microchim. Acta. 2014, 181 (1-2), 55-61.

(36) Kumar, A.; Hsu, L. H.-H.; Kavanagh, P.; Barrière, F.; Lens, P. N. L.; Lapinsonnière, L.; V, J. H. L.; Schröder, U.; Jiang, X.; Leech, D. The Ins and Outs of Microorganism-Electrode Electron Transfer Reactions. Nat. Rev. Chem. 2017, 1 (3), 0024.

(37) Mohawk, J. A.; Green, C. B.; Takahashi, J. S. Central and Peripheral Circadian Clocks in Mammals. Neuroscience 2012, 35 (1), 445-462.

(38) Pariollaud, M.; Gibbs, J. E.; Hopwood, T. W.; Brown, S.; Begley, N.; Vonslow, R.; Poolman, T.; Guo, B.; Saer, B.; Jones, D. H.; Tellam, J. P.; Bresciani, S.; Tomkinson, N. C. O.; Wojno-Picon, J.; Cooper, A. W. J.; Daniels, D. A.; Trump, R. P.; Grant, D.; Zuercher, W.; Willson, T. M.; MacDonald, A. S.; Bolognese, B.; Podolin, P. L.; Sanchez, Y.; Loudon, A. S. I.; 
Ray, D. W. Circadian Clock Component REV-ERB $\alpha$ Controls Homeostatic Regulation of Pulmonary Inflammation. J. Clin. Invest. 2018, 128 (6), 2281-2296.

(39) Woldt, E.; Sebti, Y.; Solt, L. A.; Duhem, C.; Lancel, S.; Eeckhoute, J.; Hesselink, M. K. C.; Paquet, C.; Delhaye, S.; Shin, Y.; Kamenecka, T. M.; Schaart, G.; Lefebvre, P.; Nevière, R.; Burris, T. P.; Schrauwen, P.; Staels, B.; Duez, H. Rev-Erb- $\alpha$ Modulates Skeletal Muscle Oxidative Capacity by Regulating Mitochondrial Biogenesis and Autophagy. Nat. Med. 2013, 19 (8), 1039-1046.

(40) Geldof, L.; Deventer, K.; Roels, K.; Tudela, E.; Eenoo, P. V. In Vitro Metabolic Studies of REV-ERB Agonists SR9009 and SR9011 Int. J. Mol. Sci. 2016, 17 (10), 1676.

(41) Chang, C.; Loo, C.-S.; Zhao, X.; Solt, L. A.; Liang, Y.; Bapat, S. P.; Cho, H.; Kamenecka, T. M.; Leblanc, M.; Atkins, A. R.; Yu, R. T.; Downes, M.; Burris, T. P.; Evans, R. M.; Zheng, Y. The Nuclear Receptor REV-ERB $\alpha$ Modulates Th17 Cell-Mediated Autoimmune Disease. Proc. Natl. Acad. Sci. U.S.A. 2019, 116 (37), 18528-18536.

(42) Sulli, G.; Rommel, A.; Wang, X.; Kolar, M. J.; Puca, F.; Saghatelian, A.; Plikus, M. V.; Verma, I. M.; Panda, S. Pharmacological Activation of REV-ERBs Is Lethal in Cancer and Oncogene Induced Senescence. Nature 2018, 553 (7688), 351-355.

(43) Dierickx, P.; Emmett, M. J.; Jiang, C.; Uehara, K.; Liu, M.; Adlanmerini, M.; Lazar, M. A. SR9009 Has REV-ERB-Independent Effects on Cell Proliferation and Metabolism. Proc. Natl. Acad. Sci. U.S.A. 2019, 116 (25), 12147-12152. 